Pacific

Journal of

Mathematics

SUMS OF PRODUCTS OF GENERALIZED BERNOULLI POLYNOMIALS

KWANG-Wu Chen 


\title{
SUMS OF PRODUCTS OF GENERALIZED BERNOULLI POLYNOMIALS
}

\author{
KWANG-Wu CHEN
}

In this paper, we investigate the zeta function

$$
\begin{array}{r}
Z(P, \chi, a, s)=\sum_{n_{1}=1}^{\infty} \cdots \sum_{n_{r}=1}^{\infty} \chi_{1}\left(n_{1}\right) \ldots \chi_{r}\left(n_{r}\right) \\
\cdot P\left(n_{1}+a_{1}, \ldots, n_{r}+a_{r}\right)^{-s},
\end{array}
$$

where $a_{i} \geq 0, \chi_{i}$ is a Dirichlet character with conductor $N_{i}$, and $\boldsymbol{P}$ is a polynomial satisfying certain conditions. Its special values at nonpositive integers are closely related to generalized Bernoulli polynomials. Using this fact we can easily get sums of products of Euler polynomials and generalized Bernoulli polynomials.

\section{Introduction.}

Let $\chi$ be a Dirichlet character with conductor $N$. Generalized Bernoulli numbers and polynomials are defined by Leopoldt [10] by

$$
\begin{gathered}
\sum_{k=1}^{N} \frac{\chi(k) t e^{k t}}{e^{N t}-1}=\sum_{n=0}^{\infty} \frac{B_{\chi}^{n} t^{n}}{n !}, \quad|t|<\frac{2 \pi}{N}, \\
\sum_{k=1}^{N} \frac{\chi(k) t e^{(k+x) t}}{e^{N t}-1}=\sum_{n=0}^{\infty} \frac{B_{\chi}^{n}(x) t^{n}}{n !}, \quad|t|<\frac{2 \pi}{N} .
\end{gathered}
$$

In particular, if $\chi_{0}$ is the trivial character, then

$$
\begin{aligned}
B_{\chi_{0}}^{n} & =(-1)^{n} B_{n}, & & \text { for } n \geq 0, \\
B_{\chi_{0}}^{n}(x) & =B_{n}(1+x), & & \text { for } n \geq 0 .
\end{aligned}
$$

If $\chi$ is the primitive character with conductor 4 , then

$$
B_{\chi}^{0}=0 \quad \text { and } \quad B_{\chi}^{n}=\frac{-n}{2} E_{n-1}, \quad \text { for } n \geq 1 ;
$$

(4) $\quad B_{\chi}^{0}(x)=0 \quad$ and $\quad B_{\chi}^{n}(x)=-2^{n-2} n E_{n-1}\left(\frac{x+1}{2}\right), \quad$ for $n \geq 1$. 
Let $a=\left(a_{1}, \ldots, a_{r}\right), a_{i} \geq 0, P(X)=P\left(X_{1}, \ldots, X_{r}\right)$ be a polynomial of $r$ variables with nonnegative real coefficients such that $P(n+a)>0$ for all $n \in \mathbb{N}^{r}$ and the series

$$
\sum_{n \in \mathbb{N}^{r}} P(n)^{-s}=\sum_{n_{1}=1}^{\infty} \cdots \sum_{n_{r}=1}^{\infty} P\left(n_{1}, \ldots, n_{r}\right)^{-s}
$$

is absolutely convergent for $\operatorname{Re} s>\sigma>0 . \chi_{1}, \ldots, \chi_{r}$ are nontrivial Dirichlet characters with conductors $N_{1}, \ldots, N_{r}$, respectively. Consider the zeta function

$$
\begin{aligned}
Z(P, \chi, a, s)= & \sum_{n_{1}=1}^{\infty} \cdots \sum_{n_{r}=1}^{\infty} \chi_{1}\left(n_{1}\right) \ldots \chi_{r}\left(n_{r}\right) \\
& \cdot P\left(n_{1}+a_{1}, \ldots, n_{r}+a_{r}\right)^{-s}, \quad \operatorname{Re} s>\sigma .
\end{aligned}
$$

In [2] the author and Eie considered the zeta function $Z(P, \chi, 0, s)$, and we found the special value at nonpositive integers closely related to generalized Bernoulli numbers. Using the same method as in the proof of the Main Theorem in [2], we have the following similar result for $Z(P, \chi, a, s)$ with generalized Bernoulli polynomials:

Theorem 1. $Z(P, \chi, a, s)$ defined above has a meromorphic analytic continuation to the whole complex s-plane. For any integer $m \geq 0$, if

$$
P^{m}(X)=\sum_{|\alpha|=0}^{m p} C_{\alpha} X_{1}^{\alpha_{1}} \ldots X_{r}^{\alpha_{r}}, \quad p=\operatorname{deg} P
$$

then

$$
Z(P, \chi, a,-m)=(-1)^{r} \sum_{|\alpha|=0}^{m p} C_{\alpha} \prod_{j=1}^{r} \frac{B_{\chi_{j}}^{\alpha_{j}+1}\left(a_{j}\right)}{\alpha_{j}+1} .
$$

Since Euler polynomials are special cases of generalized Bernoulli polynomials with the primitive Dirichlet character $\chi$ of conductor 4 , we can easily get the following theorem:

Theorem 2. Let $P$ and $a$ be defined as in Theorem 1. The zeta function

$$
Z(P, s)=\sum_{n_{1}=0}^{\infty} \cdots \sum_{n_{r}=0}^{\infty}(-1)^{n_{1}+\cdots+n_{r}} P\left(n_{1}+a_{1}, \ldots, n_{r}+a_{r}\right)^{-s}
$$

has a meromorphic analytic continuation to the whole complex s-plane. For any integer $m \geq 0$,

$$
Z(P,-m)=\sum_{|\alpha|=0}^{m p} C_{\alpha} \prod_{j=1}^{r} \frac{E_{\alpha_{j}}\left(a_{j}\right)}{2} .
$$


We use the notation $s(n, k)$ for the Stirling number of the first kind, the number of ways to permute a list of $n$ items into $k$ cycles (cf. [6]). Using some relations between different zeta functions and their special values at nonpositive integers, we can get sums of products of Euler polynomials, Bernoulli polynomials, and generalized Bernoulli polynomials.

Theorem 3. Let $y=x_{1}+\cdots+x_{N}$. Then

$$
\begin{aligned}
& \sum_{\substack{j_{1}+\cdots+j_{N}=m \\
j_{i} \geq 0}}\left(\begin{array}{c}
m \\
j_{1}, \ldots, j_{N}
\end{array}\right) E_{j_{1}}\left(x_{1}\right) \ldots E_{j_{N}}\left(x_{N}\right) \\
= & \frac{2^{N-1}}{(N-1) !} \sum_{k=0}^{N-1} s(N, k+1) \sum_{j=0}^{k}\left(\begin{array}{l}
k \\
j
\end{array}\right)(-y)^{k-j} E_{m+j}(y) .
\end{aligned}
$$

Theorem 4. Let $y=x_{1}+\cdots+x_{N}$. Then

$$
\begin{aligned}
& \sum_{\substack{j_{1}+\cdots+j_{N}=m \\
j_{i} \geq 0}}\left(\begin{array}{c}
m \\
j_{1}, \ldots, j_{N}
\end{array}\right) B_{j_{1}}\left(x_{1}\right) \ldots B_{j_{N}}\left(x_{N}\right) \\
= & \frac{(-1)^{N-1} m !}{(N-1) !(m-N) !} \sum_{k=0}^{N-1} s(N, k+1) \sum_{j=0}^{k}\left(\begin{array}{l}
k \\
j
\end{array}\right)(-y)^{k-j} \frac{B_{m+j-N+1}(y)}{m+j-N+1} .
\end{aligned}
$$

Theorem 5. Let $r$ be a positive integer and $\chi_{i}$ be a nontrivial Dirichlet character with conductor $N_{i}$, for $i=1,2, \ldots, r$. Then for any positive integer $m$,

$$
\begin{aligned}
& \sum_{\substack{j_{1}+\cdots+j_{r}=m \\
j_{i} \geq 0}}\left(\begin{array}{c}
m \\
j_{1}, \ldots, j_{r}
\end{array}\right) \frac{B_{\chi_{1}}^{j_{1}+1}\left(x_{1}\right)}{N_{1}^{j_{1}}\left(j_{1}+1\right)} \cdots \frac{B_{\chi_{r}}^{j_{r}+1}\left(x_{r}\right)}{N_{r}^{j_{r}}\left(j_{r}+1\right)} \\
= & \frac{-1}{(r-1) !} \sum_{a_{1}=1}^{N_{1}} \cdots \sum_{a_{r}=1}^{N_{r}} \chi_{1}\left(a_{1}\right) \ldots \chi_{r}\left(a_{r}\right) \\
& \cdot \sum_{k=0}^{r-1} s(r, k+1) \sum_{j=0}^{k}\left(\begin{array}{c}
k \\
j
\end{array}\right)(-y)^{k-j} \frac{B_{m+j+1}(y)}{m+j+1},
\end{aligned}
$$

where $y=\frac{a_{1}+x_{1}}{N_{1}}+\cdots+\frac{a_{r}+x_{r}}{N_{r}}$.

In the last section, we reproduce some classical identities among Euler polynomials using our method, and also some new identities.

\section{Sketch of proof of Theorem 1 .}

Since the proof is exactly the same as [2], we just sketch the outline. 
Finding the special value at $s=-m$ of the zeta function

$$
Z(P, \chi, a, s)=\sum_{n_{1}=1}^{\infty} \cdots \sum_{n_{r}=1}^{\infty} \chi_{1}\left(n_{1}\right) \ldots \chi_{r}\left(n_{r}\right) P(n+a)^{-s},
$$

is equivalent to finding the coefficient of $t^{m}$ in the asymptotic expansion at $t=0$ of the function

$$
\sum_{n_{1}=1}^{\infty} \cdots \sum_{n_{r}=1}^{\infty} \chi_{1}\left(n_{1}\right) \ldots \chi_{r}\left(n_{r}\right) \exp \{-P(n+a) t\} .
$$

It is also equivalent to finding the constant term in the asymptotic expansion at $t=0$ of the function

$$
g(t)=\sum_{n_{1}=1}^{\infty} \cdots \sum_{n_{r}=1}^{\infty} \chi_{1}\left(n_{1}\right) \ldots \chi_{r}\left(n_{r}\right) P^{m}(n+a) \exp \{-P(n+a) t\} .
$$

For the given polynomial

$$
P(X)=\sum_{|\alpha|=0}^{p} A_{\alpha} X^{\alpha}, \quad p=\operatorname{deg} P
$$

we let

$$
Q(X, Y)=\sum_{|\alpha|=0}^{p} A_{\alpha} X^{\alpha} Y^{p-|\alpha|}
$$

be the corresponding homogeneous polynomial in $r+1$ variables. Obviously, $Q((n+a) t, t)=P(n+a) t^{p}$ and so

$$
\begin{aligned}
g\left(t^{p}\right) & =\sum_{n \in \mathbb{N}^{r}} \chi_{1}\left(n_{1}\right) \ldots \chi_{r}\left(n_{r}\right) P^{m}(n+a) \exp \left\{-P(n+a) t^{p}\right\} \\
& =\sum_{n \in \mathbb{N}^{r}} \chi_{1}\left(n_{1}\right) \ldots \chi_{r}\left(n_{r}\right) P^{m}(n+a) \exp \{-Q((n+a) t, t)\} \\
& =\sum_{|\alpha|=0}^{m p} C_{\alpha} \sum_{n \in \mathbb{N}^{r}} \chi_{1}\left(n_{1}\right) \ldots \chi_{r}\left(n_{r}\right)(n+a)^{\alpha} \exp \{-Q((n+a) t, t)\}
\end{aligned}
$$

where

$$
P^{m}(X)=\sum_{|\alpha|=0}^{m p} C_{\alpha} X^{\alpha} \quad \text { and } \quad n^{\alpha}=n_{1}^{\alpha_{1}} \ldots n_{r}^{\alpha_{r}} .
$$

Similar to [2] we use induction on $r$ and prove that the asymptotic expansion at $t=0$ of the function

$$
f_{\beta}(t)=\sum_{n \in \mathbb{N}^{r}} \chi_{1}\left(n_{1}\right) \ldots \chi_{r}\left(n_{r}\right)(n+a)^{\beta} \exp \{-Q((n+a) t, t)\}
$$


has the form $\sum_{n=0}^{\infty} d_{n} t^{n}$ with the constant term $d_{0}$ given by

$$
d_{0}=(-1)^{r} \prod_{j=1}^{r} \frac{B_{\chi_{j}}^{\beta_{j}+1}\left(a_{j}\right)}{\beta_{j}+1} .
$$

Therefore, we get our assertion for generalized Bernoulli polynomials.

\section{Proof of Theorem 2.}

Let $\chi$ be the primitive Dirichlet character with conductor 4 . Then the zeta function can be rewritten as

$$
\begin{aligned}
Z(P, s) & =\sum_{n_{1}=0}^{\infty} \cdots \sum_{n_{r}=0}^{\infty}(-1)^{n_{1}+\cdots+n_{r}} P\left(n_{1}+a_{1}, \ldots, n_{r}+a_{r}\right)^{-s} \\
& =\sum_{n_{1}=0}^{\infty} \cdots \sum_{n_{r}=0}^{\infty} \chi\left(2 n_{1}+1\right) \ldots \chi\left(2 n_{r}+1\right) P\left(n_{1}+a_{1}, \ldots, n_{r}+a_{r}\right)^{-s} \\
& =\sum_{k_{1}=1}^{\infty} \cdots \sum_{k_{r}=1}^{\infty} \chi\left(k_{1}\right) \ldots \chi\left(k_{r}\right) P\left(\frac{k_{1}-1}{2}+a_{1}, \ldots, \frac{k_{r}-1}{2}+a_{r}\right)^{-s}
\end{aligned}
$$

Now we assume that $P^{m}(X)=\sum_{|\alpha|=0}^{m p} C_{\alpha} X^{\alpha}$, where $p=\operatorname{deg} P$. Thus

$$
\begin{aligned}
P^{m}\left(\frac{k-1}{2}+a\right) & =\sum_{|\alpha|=0}^{m p} C_{\alpha}\left(\frac{k_{1}-1}{2}+a_{1}\right)^{\alpha_{1}} \ldots\left(\frac{k_{r}-1}{2}+a_{r}\right)^{\alpha_{r}} \\
& =\sum_{|\alpha|=0}^{m p} \frac{C_{\alpha}}{2^{|\alpha|}}\left(k_{1}+2 a_{1}-1\right)^{\alpha_{1}} \ldots\left(k_{r}+2 a_{r}-1\right)^{\alpha_{r}}
\end{aligned}
$$

Now we apply Theorem 1 and Equation (4) to this zeta function

$$
\begin{aligned}
Z(P,-m) & =(-1)^{r} \sum_{|\alpha|=0}^{m p} \frac{C_{\alpha}}{2^{|\alpha|}} \prod_{j=1}^{r} \frac{B_{\chi}^{\alpha_{j}+1}\left(2 a_{j}-1\right)}{\alpha_{j}+1} \\
& =(-1)^{r} \sum_{|\alpha|=0}^{m p} \frac{C_{\alpha}}{2^{|\alpha|}} \prod_{j=1}^{r} \frac{-2^{\alpha_{j}-1}\left(\alpha_{j}+1\right) E_{\alpha_{j}}\left(a_{j}\right)}{\alpha_{j}+1} \\
& =\sum_{|\alpha|=0}^{m p} C_{\alpha} \prod_{j=1}^{r} \frac{E_{\alpha_{j}}\left(a_{j}\right)}{2} .
\end{aligned}
$$

This completes our proof. 


\section{Sums of products of Euler polynomials.}

We use a result stated in [5]. For $m_{i}$ positive integers and deg $P<m_{1}+$ $\cdots+m_{r}$, we consider the rational function

$$
F(T)=\frac{P(T)}{\left(1-T^{m_{1}}\right) \ldots\left(1-T^{m_{r}}\right)}=\sum_{k=0}^{\infty} a(k) T^{k},
$$

where $|T|<1$, and

$$
a(k)=\frac{1}{2 \pi i} \int_{C} \frac{F(z)}{z^{k+1}} d z
$$

is determined by $F$ via Cauchy's integral formula, with $C$ a sufficiently small circle centered at the origin and going counterclockwise. The zeta function (cf. Chapter XVII of [8])

$$
Z_{F}(s)=\sum_{k=1}^{\infty} a(k) k^{-s}
$$

is related to $F(T)$ via a Mellin transform

$$
Z_{F}(s) \Gamma(s)=\int_{0}^{\infty} t^{s-1}\left[F\left(e^{-t}\right)-F(0)\right] d t,
$$

for Re $s$ sufficiently large. The main tool that we use to prove the following theorems and propositions is as follows:

Lemma (Lemma 3 of [5]). Given

$$
P(T)=\sum_{j=0}^{m} b_{j} T^{j} \quad \text { and } \quad F(T)=\frac{P(T)}{\left(1-T^{m_{1}}\right) \ldots\left(1-T^{m_{r}}\right)}
$$

with $m_{1}+\cdots+m_{r}>m$, then, for $|T|<1$ we have

$$
F(T)=\sum_{j=0}^{m} b_{j} \sum_{n_{1}=0}^{\infty} \cdots \sum_{n_{r}=0}^{\infty} T^{n_{1} m_{1}+\cdots+n_{r} m_{r}+j}
$$

and hence the associated zeta function

$$
\begin{aligned}
Z_{F}(s)= & b_{0} \sum_{\substack{n_{1}, \ldots, n_{r} \geq 0 \\
|n|>0}}\left(n_{1} m_{1}+\cdots+n_{r} m_{r}\right)^{-s} \\
& +\sum_{j=1}^{m} b_{j} \sum_{n_{1}, \ldots, n_{r} \geq 0}\left(n_{1} m_{1}+\cdots+n_{r} m_{r}+j\right)^{-s} .
\end{aligned}
$$


Using the above statements we can prove Theorem 3. Consider the rational function

$$
\begin{aligned}
F(T) & =\frac{T^{x_{1}}}{1+T} \ldots \frac{T^{x_{N}}}{1+T} \\
& =\left[T^{x_{1}} \sum_{n_{1}=0}^{\infty}(-T)^{n_{1}}\right] \ldots\left[T^{x_{N}} \sum_{n_{N}=0}^{\infty}(-T)^{n_{N}}\right] \\
& =\sum_{n_{1}=0}^{\infty} \cdots \sum_{n_{N}=0}^{\infty}(-1)^{n_{1}+\cdots+n_{N}} T^{\left(n_{1}+x_{1}\right)+\cdots+\left(n_{N}+x_{N}\right)} .
\end{aligned}
$$

Its associated zeta function is

$$
Z_{F}(s)=\sum_{n_{1}=0}^{\infty} \cdots \sum_{n_{N}=0}^{\infty}(-1)^{n_{1}+\cdots+n_{N}}\left[\left(n_{1}+x_{1}\right)+\cdots+\left(n_{N}+x_{N}\right)\right]^{-s} .
$$

Using the result of Theorem 2 we know that for $m \geq 0$,

$$
Z_{F}(-m)=2^{-N} \sum_{\substack{j_{1}+\cdots+j_{N}=m \\
j_{i} \geq 0}}\left(\begin{array}{c}
m \\
j_{1}, \ldots, j_{N}
\end{array}\right) E_{j_{1}}\left(x_{1}\right) \ldots E_{j_{N}}\left(x_{N}\right) .
$$

On the other hand, let $y=x_{1}+\cdots+x_{N}$; we can rewrite the rational function $F(T)$ as

$$
F(T)=\frac{T^{y}}{(1+T)^{N}}=\sum_{n=0}^{\infty}(-1)^{n} \frac{(n+N-1)(n+N-2) \ldots(n+1)}{(N-1) !} T^{n+y} .
$$

The associated zeta function can also be rewritten as

$$
\begin{aligned}
Z_{F}(s) & =\sum_{n=0}^{\infty} \frac{(-1)^{n}}{(N-1) !}(n+N-1)(n+N-2) \ldots(n+1)(n+y)^{-s} \\
& =\sum_{n=0}^{\infty} \frac{(-1)^{n}}{(N-1) !} \sum_{k=0}^{N-1} s(N, k+1) n^{k} \cdot(n+y)^{-s},
\end{aligned}
$$

since (cf. Eq. (7.48) of [6])

$$
(n+1)(n+2) \ldots(n+N-1)=\sum_{k=0}^{N-1} s(N, k+1) n^{k} .
$$

Thus

$$
\begin{aligned}
Z_{F}(s) & =\sum_{n=0}^{\infty} \frac{(-1)^{n}}{(N-1) !} \sum_{k=0}^{N-1} s(N, k+1)(n+y-y)^{k} \cdot(n+y)^{-s} \\
& =\sum_{n=0}^{\infty} \frac{(-1)^{n}}{(N-1) !} \sum_{k=0}^{N-1} s(N, k+1) \sum_{j=0}^{k}\left(\begin{array}{c}
k \\
j
\end{array}\right)(-y)^{k-j}(n+y)^{j-s}
\end{aligned}
$$


Again using the result of Theorem 2 (this time for $r=1$ and $P(x)=x$ ) we know that for $m \geq 0$

$$
Z_{F}(-m)=\sum_{k=0}^{N-1} \frac{s(N, k+1)}{(N-1) !} \sum_{j=0}^{k}\left(\begin{array}{l}
k \\
j
\end{array}\right)(-y)^{k-j} \frac{E_{m+j}(y)}{2} .
$$

Now combine Equation (10) and Equation (11) to obtain our assertion.

\section{Sums of products of generalized Bernoulli polynomials.}

We first prove Theorem 4, then apply it to prove Theorem 5. The proof of Theorem 4 is similar to the proof of Theorem 3. We just consider the different rational function

$$
F(T)=\frac{T^{x_{1}}}{1-T} \cdots \frac{T^{x_{N}}}{1-T}=\sum_{n_{1}=0}^{\infty} \cdots \sum_{n_{N}=0}^{\infty} T^{\left(n_{1}+x_{1}\right)+\cdots+\left(n_{N}+x_{N}\right)} .
$$

Its associated zeta function is

$$
Z_{F}(s)=\sum_{n_{1}=0}^{\infty} \cdots \sum_{n_{N}=0}^{\infty}\left[\left(n_{1}+x_{1}\right)+\cdots+\left(n_{N}+x_{N}\right)\right]^{-s}
$$

Using the result of Proposition 2 in [5], we know that for $m \geq 0$

$$
Z_{F}(-m)=(-1)^{N} \sum_{\substack{j_{1}+\cdots+j_{N}=m+N \\
j_{i} \geq 0}}\left(\begin{array}{c}
m \\
j_{1}, \ldots, j_{N}
\end{array}\right) B_{j_{1}}\left(x_{1}\right) \ldots B_{j_{N}}\left(x_{N}\right) .
$$

On the other hand, let $y=x_{1}+\cdots+x_{N}$; we can rewrite the rational function $F(T)$ as

$$
F(T)=\frac{T^{y}}{(1-T)^{N}}=\sum_{n=0}^{\infty} \frac{T^{n+y}}{(N-1) !} \sum_{k=0}^{N-1} s(N, k+1) n^{k} .
$$

The associated zeta function can also rewrite as

$$
Z_{F}(s)=\sum_{n=0}^{\infty} \frac{1}{(N-1) !} \sum_{k=0}^{N-1} s(N, k+1) \sum_{j=0}^{k}\left(\begin{array}{l}
k \\
j
\end{array}\right)(-y)^{k-j}(n+y)^{j-s} .
$$

Again using the same result of Proposition 2 in [5], we have for $m \geq 0$

$$
Z_{F}(-m)=\sum_{k=0}^{N-1} \frac{s(N, k+1)}{(N-1) !} \sum_{j=0}^{k}\left(\begin{array}{l}
k \\
j
\end{array}\right)(-y)^{k-j} \frac{-B_{m+j+1}(y)}{m+j+1} .
$$

Now combine Equation (12), Equation (13), and change $m+N$ to $m$, to conclude the proof of Theorem 4 . 
To prove Theorem 5 , we consider the zeta function

$$
Z(s)=\sum_{n_{1}=1}^{\infty} \ldots \sum_{n_{r}=1}^{\infty} \chi_{1}\left(n_{1}\right) \ldots \chi_{r}\left(n_{r}\right)\left(\sum_{j=1}^{r}\left(\prod_{\substack{i=1 \\ i \neq j}}^{r} N_{i}\right)\left(n_{j}+x_{j}\right)\right)^{-s} .
$$

Substitute $n_{i}=a_{i}+N_{i} m_{i}$ where $a_{i}=1, \ldots, N_{i}$ and $m_{i} \geq 0$ for $i=1, \ldots, r$. Thus $Z(s)$ becomes

$$
\sum_{a_{1}=1}^{N_{1}} \ldots \sum_{a_{r}=1}^{N_{r}} \sum_{m_{1}=0}^{\infty} \cdots \sum_{m_{r}=0}^{\infty}\left(\prod_{i=1}^{r} \chi_{i}\left(a_{i}+m_{i} N_{i}\right) N_{i}^{-s}\right)\left[\sum_{j=1}^{r}\left(m_{j}+\frac{a_{j}+x_{j}}{N_{j}}\right)\right]^{-s} .
$$

Now we let

$$
Z_{B}(s)=\sum_{m_{1}=0}^{\infty} \cdots \sum_{m_{r}=0}^{\infty}\left(\prod_{i=1}^{r} N_{i}\right)^{-s}\left[\sum_{j=1}^{r}\left(m_{j}+\frac{a_{j}+x_{j}}{N_{j}}\right)\right]^{-s} .
$$

Then we can represent the zeta function $Z(s)$ as

$$
Z(s)=\sum_{a_{1}=1}^{N_{1}} \cdots \sum_{a_{r}=1}^{N_{r}}\left(\prod_{i=1}^{r} \chi_{i}\left(a_{i}\right)\right) Z_{B}(s)
$$

From [4] we know that this zeta function $Z_{B}(s)$ has an analytic continuation to the whole complex plane, and the special values at nonpositive integers $s=-m$ are given by

$$
Z_{B}(-m)=\left(\prod_{i=1}^{r} N_{i}^{m}\right) \sum_{\substack{p_{1}+\cdots+p_{r}=m+r \\ p_{1}, \ldots, p_{r} \geq 0}} \frac{m !}{p_{1} ! \ldots p_{r} !} \prod_{j=1}^{r} B_{p_{j}}\left(\frac{a_{j}+x_{j}}{N_{j}}\right) .
$$

Using the result of Theorem 4 we can rewrite $Z_{B}(-m)$ as

$$
\frac{\left(\prod_{i=1}^{r} N_{i}^{m}\right)(-1)^{r-1}}{(r-1) !} \sum_{k=0}^{r-1} s(r, k+1) \sum_{j=0}^{k}\left(\begin{array}{l}
k \\
j
\end{array}\right)(-y)^{k-j} \frac{B_{m+j+1}(y)}{m+j+1},
$$

where $y=\frac{a_{1}+x_{1}}{N_{1}}+\cdots+\frac{a_{r}+x_{r}}{N_{r}}$. Now applying Theorem 1 , the special values at nonpositive integers $s=-m$ of the zeta function $Z(s)$ are

$$
Z(-m)=\sum_{\substack{p_{1}+\ldots+p_{r}=m \\
p_{1}, \ldots, p_{r} \geq 0}}\left(\begin{array}{c}
m \\
p_{1}, \ldots, p_{r}
\end{array}\right)(-1)^{r}\left(\prod_{i=1}^{r} \frac{N_{i}^{m-p_{i}} B_{\chi_{i}}^{p_{i}+1}\left(x_{i}\right)}{p_{i}+1}\right) .
$$

On the other hand, using the equality

$$
Z(-m)=\sum_{a_{1}=1}^{N_{1}} \cdots \sum_{a_{r}=1}^{N_{r}} \prod_{i=1}^{r} \chi_{i}\left(a_{i}\right) Z_{B}(-m)
$$

and the above values of $Z(-m)$ and $Z_{B}(-m)$, we get our assertion. 


\section{Remark.}

(1) Dilcher in [3] produced Equations (7) and (8) in a different way. These formulae are the same, except for the definition of the Stirling numbers of the first kind.

(2) The author and Eie in [2] produced a formula with sums of products of generalized Bernoulli numbers; here we have used the same ideas to prove a similar formula with generalized Bernoulli polynomials.

(3) Huang and Huang in [9] gave some generalized formulas for sums of products of Bernoulli numbers and polynomials via a different method called algebraic residues.

\section{Some further identities.}

Applying the method of proof of Theorems 3 and 4 to different rational functions, we can get different identities between generalized Bernoulli polynomials, Euler polynomials, and Bernoulli polynomials. Here we list some classical identities among Euler polynomials (cf. [1]).

Proposition 1 (see 23.1.7 of [1]).

$$
E_{m}(x+h)=\sum_{k=0}^{m}\left(\begin{array}{c}
m \\
k
\end{array}\right) E_{k}(x) h^{m-k}=\sum_{k=0}^{m}\left(\begin{array}{c}
m \\
k
\end{array}\right) E_{k}(h) x^{m-k},
$$

for any nonnegative integer $m$.

Proof. Consider the zeta function

$$
Z(P, s)=\sum_{n=0}^{\infty}(-1)^{n}(n+x+h)^{-s}, \quad \text { where } P^{m}(z)=(z+x+h)^{m} .
$$

We can express $P^{m}(z)$ in a different way, as

$$
P^{m}(z)=\sum_{k=0}^{m}\left(\begin{array}{c}
m \\
k
\end{array}\right)(z+x)^{k} h^{m-k}, \quad \text { or } \sum_{k=0}^{m}\left(\begin{array}{c}
m \\
k
\end{array}\right)(z+h)^{k} x^{m-k} .
$$

Then we apply Theorem 2 to this zeta function $Z(P, s)$ to obtain the assertion.

Proposition 2 (see 23.1 .10 of [1] ).

$$
E_{m}(k x)= \begin{cases}k^{m} \sum_{i=0}^{k-1}(-1)^{i} E_{m}\left(x+\frac{i}{k}\right), & \text { if } k \text { is odd, } \\ -\frac{2}{m+1} k^{m} \sum_{i=0}^{k-1}(-1)^{i} B_{m+1}\left(x+\frac{i}{k}\right), & \text { if } k \text { is even, }\end{cases}
$$

for any nonnegative integer $m$.

Proof. We consider the zeta function

$$
Z(P, s)=\sum_{n=0}^{\infty}(-1)^{n}(n+k x)^{-s}=\sum_{n=0}^{\infty} \sum_{i=0}^{k-1}(-1)^{n k+i}(n k+i+k x)^{-s} .
$$


Now we separate $k$ into two cases, odd and even.

$$
Z(P, s)= \begin{cases}\sum_{i=0}^{k-1}(-1)^{i} k^{-s} \sum_{n=0}^{\infty}(-1)^{n}\left(n+x+\frac{i}{k}\right)^{-s}, & \text { if } k \text { is odd } \\ \sum_{i=0}^{k-1}(-1)^{i} k^{-s} \sum_{n=0}^{\infty}\left(n+x+\frac{i}{k}\right)^{-s}, & \text { if } k \text { is even }\end{cases}
$$

Again we apply Theorem 2 to $Z(P, s)$ and complete the proof.

Proposition 3 (see Eq. (51.6.5) of [7]). For any nonnegative integer $m$,

$$
\begin{aligned}
& \sum_{k=0}^{m}\left(\begin{array}{c}
m \\
k
\end{array}\right) 2^{k} E_{m-k}(x) E_{k}(y) \\
& =E_{m}(x+2 y)+2^{m} E_{m}\left(\frac{x+2 y}{2}\right)-2^{m} E_{m}\left(\frac{x+2 y+1}{2}\right) .
\end{aligned}
$$

Proof. Follow a similar argument as in the proof of the previous proposition, but consider the different fraction

$$
\begin{aligned}
F(T) & =4 \cdot \frac{T^{2 x}}{1+T^{2}} \cdot \frac{T^{4 y}}{1+T^{4}} \\
& =2 \cdot \frac{T^{2 x+4 y}}{1+T^{2}}+2 \cdot \frac{T^{2 x+4 y}}{1+T^{4}}-2 \cdot \frac{T^{2 x+4 y+2}}{1+T^{4}} .
\end{aligned}
$$

From the associated zeta functions, we get the identity

$$
\begin{aligned}
& 4 \sum_{n_{1}=0}^{\infty} \sum_{n_{2}=0}^{\infty}(-1)^{n_{1}+n_{2}}\left[\left(2 n_{1}+2 x\right)+\left(4 n_{2}+4 y\right)\right]^{-s} \\
& =2 \sum_{n=0}^{\infty}(-1)^{n}(2 n+2 x+4 y)^{-s}+2 \sum_{n=0}^{\infty}(-1)^{n}(4 n+2 x+4 y)^{-s} \\
& \quad-2 \sum_{n=0}^{\infty}(-1)^{n}(4 n+2 x+4 y+2)^{-s} .
\end{aligned}
$$

Then we calculate the special value of $s=-m$ with $m \geq 0$ and obtain Proposition 3.

The following results give new identities for Euler polynomials:

Proposition 4. Let a be any positive odd integer. Then for any nonnegative integer $m$, we have: 
(1) If $a=4 k+1$, then

$$
\begin{aligned}
& \sum_{l=0}^{m}\left(\begin{array}{c}
m \\
l
\end{array}\right) 2^{l} E_{l}(x) a^{m-l} E_{m-l}(y) \\
& =\frac{1}{a} E_{m}(2 x+a y)+2^{m} E_{m}\left(\frac{2 x+a y}{2}\right)-2^{m} E_{m}\left(\frac{2 x+a y+1}{2}\right) \\
& \quad+\sum_{n=1}^{k}\left[a^{m-1}(a-1) E_{m}\left(\frac{2 x+a y+4 n}{a}\right)\right. \\
& \quad-a^{m-1}(a-1) E_{m}\left(\frac{2 x+a y+4 n-1}{a}\right) \\
& \quad-a^{m-1}(a+1) E_{m}\left(\frac{2 x+a y+4 n-2}{a}\right) \\
& \left.\quad+a^{m-1}(a+1) E_{m}\left(\frac{2 x+a y+4 n-3}{a}\right)\right] \\
& +a^{m-1}(a-1) E_{m}\left(\frac{2 x+a y}{a}\right)
\end{aligned}
$$

(2) If $a=4 k+3$, then

$$
\begin{aligned}
& \sum_{l=0}^{m}\left(\begin{array}{c}
m \\
l
\end{array}\right) 2^{l} E_{l}(x) a^{m-l} E_{m-l}(y) \\
& =\frac{1}{a} E_{m}(2 x+a y)+2^{m} E_{m}\left(\frac{2 x+a y}{2}\right)+2^{m} E_{m}\left(\frac{2 x+a y+1}{2}\right) \\
& \quad-\left\{\sum _ { n = 1 } ^ { k } \left[a^{m-1}(a+1) E_{m}\left(\frac{2 x+a y+4 n+2}{a}\right)\right.\right. \\
& \quad+a^{m-1}(a-1) E_{m}\left(\frac{2 x+a y+4 n+1}{a}\right) \\
& \quad-a^{m-1}(a-1) E_{m}\left(\frac{2 x+a y+4 n}{a}\right) \\
& \left.\quad-a^{m-1}(a+1) E_{m}\left(\frac{2 x+a y+4 n-1}{a}\right)\right] \\
& \quad+a^{m-1}(a+1) E_{m}\left(\frac{2 x+a y+2}{a}\right)+a^{m-1}(a-1) E_{m}\left(\frac{2 x+a y+1}{a}\right) \\
& \left.\quad-a^{m-1}(a-1) E_{m}\left(\frac{2 x+a y}{a}\right)\right\} .
\end{aligned}
$$


Proof. The proof is similar to that of Proposition 3, but with

$$
\begin{aligned}
F(T)= & 4 \cdot \frac{T^{2 x}}{1+T^{2}} \cdot \frac{T^{a y}}{1+T^{a}} \\
= & \frac{2}{a} \frac{T^{2 x+a y}}{1+T}+2 \frac{T^{2 x+a y}}{1+T^{2}}-2 \frac{T^{2 x+a y+1}}{1+T^{2}} \\
& +\frac{2}{1+T^{a}}\left\{\sum _ { n = 1 } ^ { k } \left[\frac{a-1}{a} T^{2 x+a y+4 n}-\frac{a-1}{a} T^{2 x+a y+4 n-1}\right.\right. \\
& \left.\left.\quad-\frac{a+1}{a} T^{2 x+a y+4 n-2}+\frac{a+1}{a} T^{2 x+a y+4 n-3}\right]+\frac{a-1}{a} T^{2 x+a y}\right\},
\end{aligned}
$$

for $a=4 k+1$ and

$$
\begin{aligned}
F(T)= & \cdot \frac{T^{2 x}}{1+T^{2}} \cdot \frac{T^{a y}}{1+T^{a}} \\
= & \frac{2}{a} \frac{T^{2 x+a y}}{1+T}+2 \frac{T^{2 x+a y}}{1+T^{2}}+2 \frac{T^{2 x+a y+1}}{1+T^{2}} \\
- & \frac{2}{1+T^{a}}\left\{\sum _ { n = 1 } ^ { k } \left[\frac{a+1}{a} T^{2 x+a y+4 n+2}\right.\right. \\
& \left.+\frac{a-1}{a} T^{2 x+a y+4 n+1}-\frac{a-1}{a} T^{2 x+a y+4 n}-\frac{a+1}{a} T^{2 x+a y+4 n-1}\right] \\
& \left.+\frac{a+1}{a} T^{2 x+a y+2}+\frac{a-1}{a} T^{2 x+a y+1}-\frac{a-1}{a} T^{2 x+a y}\right\},
\end{aligned}
$$

for $a=4 k+3$, respectively.

Remark. We can generalize the previous propositions to formulas involving $a^{k}$ and $b^{m-k}$ for arbitrary integers $a$ and $b$, depending on a suitable partial fraction decomposition of the function $F(T)$.

Acknowledgements. The author would like to thank the referee for some useful comments and suggestions. And this paper was supported by the National Science Council of R. O. C. grant under NSC 89-2115-M-122-006 and the Yuanpei University of Science and Technology.

\section{References}

[1] M. Abramowitz and I.A. Stegun, Handbook of Mathematical Functions with Formulas, Graphs, and Mathematical Tables, Dover Publications, Inc., New York, 1972, MR 85j:00005a, Zbl 0543.33001.

[2] K.-W. Chen and M. Eie, A note on generalized Bernoulli numbers, Pacific J. Math., 199(1) (2001), 41-59, MR 2002k:11148. 
[3] K. Dilcher, Sums of products of Bernoulli numbers, J. Number Theory, 60 (1996), 23-41, MR 97h:11014, Zbl 0863.11011.

[4] M. Eie and K.-W. Chen, A theorem on zeta functions associated with polynomials, Trans. Amer. Math. Soc., 351 (1999), 3217-3228, MR 99m:11099, Zbl 0928.11038.

[5] M. Eie and K.F. Lai, On Bernoulli identities and applications, Rev. Mat. Iberoamericana, 14(1) (1998), 167-213, MR 99h:11017.

[6] R. Graham, D. Knuth and O. Patashnik, Concrete Mathematics, Addison-Wesley, 1989, MR 91f:00001, Zbl 0668.00003.

[7] E.R. Hansen, A Table of Series and Products, Prentice-Hall, Englewood Cliffs, NJ, 1975, CMP 8567 18, Zbl 0438.00001.

[8] G.H. Hardy and E.M. Wright, An Introduction to the Theory of Numbers, Oxford University Press, 5-th edition, 1979, MR 81i:10002, Zbl 0423.10001.

[9] I.-C. Huang and S.-Y. Huang, Bernoulli numbers and polynomials via residues, J. Number Theory, 76 (1999), 178-193, MR 2000d:11027, Zbl 0940.11009.

[10] H.W. Leopoldt, Eine verallgemeinerung der Bernoullischen zahlen, Abh. Math. Sem. Univ. Hamburg, 22 (1958), 131-140, MR 19,1161e, Zbl 0080.03002.

Received May 9, 2001 and revised November 13, 2001.

Department of International Trade

Ching-Yun Institute of Technology

Jungli City, TaOyuan 320, Taiwan

Republic OF ChinA

E-mail address: kwchen@cyit.edu.tw 